\title{
In Situ Investigation of the Early-Stage Growth of Nanoporous Alumina
}

\author{
Thérèse Gorisse, ${ }^{1}$ Ludovic Dupré, ${ }^{1}$ Marc Zelsmann $\left(D,{ }^{2}\right.$ Alina Vlad, ${ }^{3}$ Alessandro Coati, ${ }^{3}$ \\ Yves Garreau, ${ }^{3,4}$ and Denis Buttard iD ${ }^{1,5}$ \\ ${ }^{1}$ Institut Nanoscience et Cryogénie-CEA, 17 avenue des Martyrs, 38000 Grenoble, France \\ ${ }^{2}$ Laboratoire des Technologies de la Microélectronique-CNRS, 17 avenue des Martyrs, 38000 Grenoble, France \\ ${ }^{3}$ Synchrotron SOLEIL, L'Orme des Merisiers, Saint-Aubin, 91192 Gif-sur-Yvette, France \\ ${ }^{4}$ Matériaux et Phénomènes Quantiques (MPQ), Université Paris Diderot-Paris 7, Sorbonne Paris Cité, CNRS, UMR 7162, 10, \\ 75205 Paris Cedex 13, France \\ ${ }^{5}$ Université Grenoble Alpes, IUT1, 17 quai Claude Bernard 38000 Grenoble, France
}

Correspondence should be addressed to Denis Buttard; denis.buttard@cea.fr

Received 14 February 2018; Accepted 26 July 2018; Published 17 October 2018

Academic Editor: William Yu

Copyright ( 92018 Thérèse Gorisse et al. This is an open access article distributed under the Creative Commons Attribution License, which permits unrestricted use, distribution, and reproduction in any medium, provided the original work is properly cited.

We report the successful use of in situ grazing incidence small-angle X-ray scattering to follow the anodization of aluminum. A dedicated electrochemical cell was designed and developed for this purpose with low X-ray absorption, with the possibility to access all azimuthal angles $\left(360^{\circ}\right)$ and to remotely control the temperature of the electrolyte. Three well-known fabrication techniques of nanoporous alumina, i.e., single, double, and pretextured, were investigated. The differences in the evolution of the scattering images are described and explained. From these measurements, we could determine at which moment the pores start growing even for very short anodization times. Furthermore, we could follow the thickness of the alumina layer as a function of the anodization time by monitoring the period of the Kiessig fringes. This work is aimed at helping to understand the different steps taking place during the anodization of aluminum at the very early stages of nanoporous alumina formation.

\section{Introduction}

With the recent rise of nanotechnologies, the development of many devices critically depends on the ability to control the synthesis of new nanoobject architectures, for example, nanowires or nanotubes arrays $[1,2]$. In this context, the directed self-assembly (DSA) fabrication technique seems to be very promising for various applications such as photonics or materials for energy applications and health [3]. Anodic aluminum oxide (AAO) is known for a long time, but regained interest over the last decades as a promising template for DSA [4]. AAO is obtained by an electrochemical oxidation of aluminum in acidic solutions. A highly dense, roughly triangular array of nanopores is then produced in the alumina layer $[5,6]$. By varying experimental parameters such as the electrolyte, the applied voltage, or the anodization time, the geometrical characteristics of the porous membrane can be adjusted. In particular, the diameter, the height of pores, and the distance between nearest neighbors can be tuned. The AAO presents the advantage of very high aspect ratio features with no real limitation. Besides, due to its thermal and mechanical characteristics, the AAO matrix allows additional processing steps, necessary for the integration into functional devices. Consequently, this matrix is a good candidate for the elaboration of organic, inorganic, or metallic dense nanowire/nanotube arrays [7].

To improve the control of the formation of AAO arrays, various top-down methods have been proposed in the literature to prepattern the aluminum surface prior to the electrochemical treatment. Among them, focused ion beam lithography 
[8], holographic lithography [9], block copolymer micelles [10], soft imprinting [11], mould-assisted chemical etching [12], colloidal lithography [13], nanoindentation [14], nanoimprint lithography (NIL) $[15,16]$, and guided electric field [17] can be cited. Such approaches are not only very interesting in terms of pores positioning and control of pore's size distribution but also allow the use of a micrometer-thick initial aluminum layer supported by a silicon wafer. Among all top-down guiding methods, NIL is very promising since it is the only approach that allows working with perfectly organized arrays on large areas at a reasonable cost. Though it is generally prepared with expensive exposure tools like electron beam lithography, the mould can be reused numerous times. Also, compared to nanoindentation, the use of an intermediate resin transfer layer permits to work with fragile substrates. At last, NIL is perfectly adapted to the already existing microelectronic processing tools.

Some fundamental questions remain regarding the pore formation and organization of AAO, such as the initial time of pore creation with the relating pore shape and geometry at the early stages, which are of paramount importance in the currently realization of high-tech devices of high quality. In this respect, the structural investigations over large samples areas are mandatory especially at the early stages of formation. Grazing incidence X-ray scattering techniques are well adapted to perform such analysis, because of their ability to study buried structures and interfaces. In particular, grazing incidence small-angle X-ray scattering (GISAXS) has been developed [18] for the analysis of nanoobjects on a large surface with a good statistical relevance. With in situ GISAXS observation during the electrochemical process, we obtained a powerful tool to investigate the early stages of pore formation and learn more about the kinetics of the pore nucleation in terms of dimension, spacing, shape, and about the evolution during the pore growth.

In this article, we present an in situ structural investigation of porous alumina template using GISAXS, where the structure evolution is followed during anodization. Three fabrication techniques are compared: single, double, and pretextured anodization via nanoimprint lithography. Morphologic parameters such as pore diameter and length are investigated. Particularly, we accurately observed the early stages of pore formation. The characteristic of the pore array structure is also measured and analyzed.

\section{Experimental}

Nanoporous alumina is achieved following an electrochemical process, which consists in the anodization of an aluminum layer in an acid bath. In this study, three fabrication techniques of nanoporous alumina are investigated: the single anodization, the double anodization, and the single anodization after the pretexturization of the aluminum layer. Each technique requires different sample preparations. For each type of technique, aluminum is deposited on a highly doped silicon wafer (resistivity $\rho=0.01 \Omega \mathrm{cm}$ ) using an electron beam evaporator in a high vacuum chamber. The thickness of the layer depends on the anodization techniques: $10 \mu \mathrm{m}$ for the single or the double anodization and
$1 \mu \mathrm{m}$ for the pretexturization technique. Samples dedicated to the single anodization are then ready to be anodized during the X-ray measurements. For the double anodization, samples are first anodized in a standard electrochemical cell until one-third of the initial Al thickness is oxidized. During this first step, the pores slowly organized in a hexagonal structure. This layer is then etched in an orthophosphoric acid bath. The bottom shape of the pores of the first anodization will guide the pores growth in the second anodization. By this way, the pores will be organized in domains of hexagonal array $[3,7,8]$. The second anodization will be performed during the X-ray measurements and at the same voltage as the first anodization. For pretextured samples, thermal nanoimprint lithography (NIL) is used to pattern the aluminum surface. The technique employed is the same as the one described in our previous paper [11]. The final sample is an aluminum layer patterned with holes of a few nanometers in depth organized in a regular quasihexagonal array. Pretextured samples are then ready to be anodized during the GISAXS measurements at the voltage corresponding to the lattice of the imprinted pattern. Prior to the X-ray measurement samples were observed ex situ with a ZEISS ultra 55 (Carl Zeiss, Germany) scanning electron microscope (SEM). As showed in Figure 1, the porous alumina is characterized by the height $h$ of the pores, the period $a$ of the porous array, and the domain size $\Delta d$ (area with a coherent hexagonal order).

The GISAXS measurements were performed at the SixS beamline of the Synchrotron SOLEIL in Saint-Aubin (Paris, France) using a photon energy of $18.4 \mathrm{keV}$. Samples were mounted on a $2+3$ circle diffractometer [19], and a Rayonix SX-165 CCD detector with $80 \times 80 \mu \mathrm{m}^{2}$ pixels was used to acquire the GISAXS data. As porous structures have periods up to $250 \mathrm{~nm}$, the scattering signals are very close to the origin of the reciprocal space. For this reason, the camera has been placed $9 \mathrm{~m}$ away from the sample, in an adjacent experimental hutch. The X-ray beam had to pass through a small hole of $1 \mathrm{~cm}$ diameter in the wall between the two experimental hutches. A beam stop was used to mask the specular reflected and direct beams. The GISAXS images were processed using the ImageJ software to improve the visualization (color, logarithmic scale, etc.). A schematic view of the GISAXS experimental setup is presented in Figure 2(a). An incident X-ray beam of wave vector $\overrightarrow{k_{i}}$ is impinging on the sample with a very low incidence angle $\alpha_{i}$ (close to the critical angle $\alpha_{c}$ ). After its interaction with the sample, the beam is transmitted, reflected, and refracted with a wave vector $\overrightarrow{k_{f}}$ which has an out-of-plane angle $\alpha_{f}$ and an inplane angle $2 \theta_{f}$ with respect to the incident beam.

The azimuth angle $\mu$ corresponds to the inplane rotation of the sample. In the reciprocal space, the scattering vector $\vec{q}$ is defined by the following equation [18]:

$$
\vec{q}=\overrightarrow{k_{f}}-\overrightarrow{k_{i}}
$$

with the wave vector modulus: $\left|k_{f}\right|=\left|k_{i}\right|=k_{0}=(2 \pi) /(\lambda)$. 


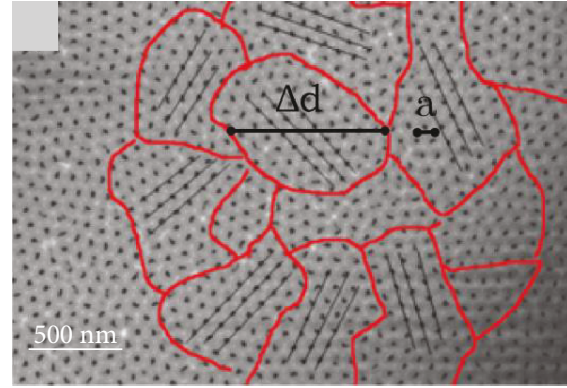

(a)

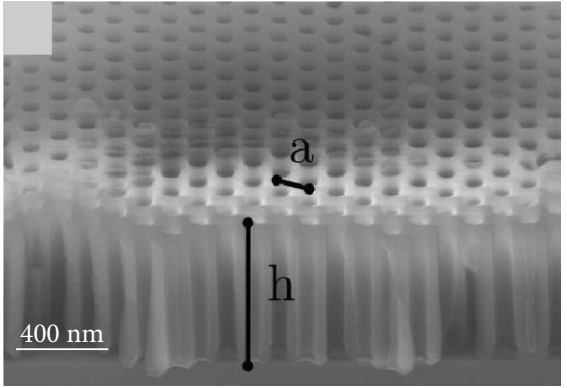

(b)

FIGURE 1: Ex situ scanning electron microscopy images of nanoporous alumina structure (a) plane view in the case of a double anodization at $40 \mathrm{~V}$ with oxalic at $3 \mathrm{wt} \%$ and (b) cross section in the case of sample pretextured by nanoimprint lithography followed by an anodization in phosphoric acid $5 \mathrm{wt} \%$ under $100 \mathrm{~V}$.

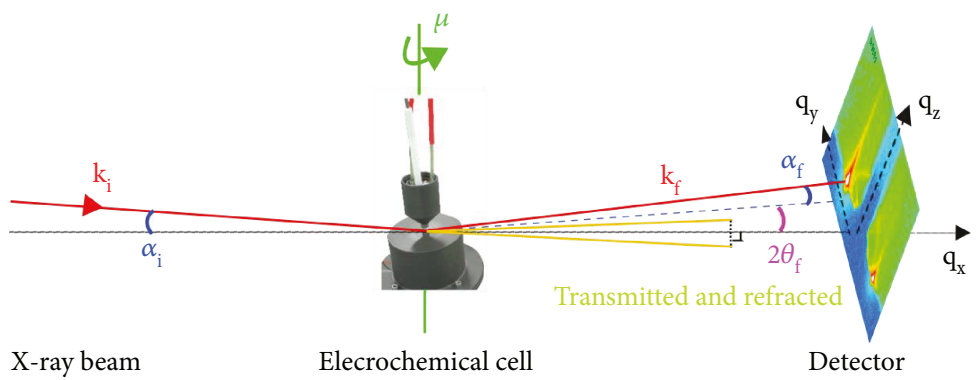

(a)

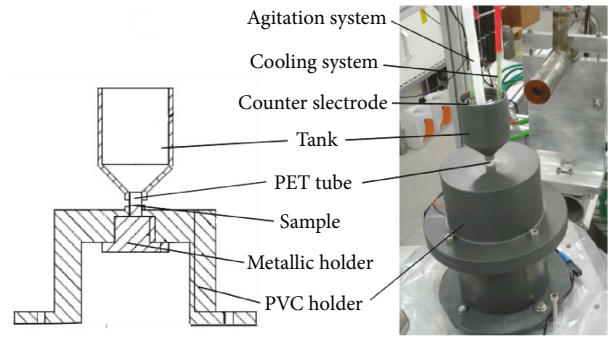

(b)

FIGURE 2: (a) Scheme of the GISAXS setup, with $\alpha_{i}$, the incidence angle; $\alpha_{f}$, the outplane emergence angle; $2 \theta_{f}$, the inplane emergence angle; $k_{i}$ and $k_{f}$, the incident and scattering wave vector; $\mu$, the rotation angle of the sample; and $q_{x}, q_{y}$, and $q_{z}$, the coordinates of the scattering vector. (b) Scheme and image of the electrochemical cell used for in situ GISAXS experiments.

The components $q_{x}, q_{y}$, and $q_{z}$ of the scattering vector are expressed as follows:

$$
\left(\begin{array}{c}
q_{x}=k_{0}\left[\cos \left(\alpha_{f}\right) \cos \left(2 \theta_{f}\right)-\cos \left(\alpha_{i}\right)\right] \\
q_{x}=k_{0}\left[\cos \left(\alpha_{f}\right) \sin \left(2 \theta_{f}\right)\right] \\
q_{z}=k_{0}\left[\sin \left(\alpha_{f}\right)+\sin \left(\alpha_{i}\right)\right]
\end{array}\right),
$$

which gives for small angles as follows:

$$
\left(\begin{array}{c}
q_{x} \approx 0 \\
q_{y} \approx 2 k_{0} \theta_{f} \\
q_{z} \approx k_{0}\left(\alpha_{f}+\alpha_{i}\right)
\end{array}\right) .
$$

An electrochemical cell was specifically designed for the GISAXS measurements (Figure 2(b)). This new cell consists of an upper tank in which the electrolyte, the agitation system, the cooling system, and the counter electrode are placed. In addition, the base of the tank around the sample is made of a polyethylene terephthalate (PET) tube of $8.7 \mathrm{~mm}$ inner diameter and $1 \mathrm{~mm}$ thick walls. PET material was specifically chosen because of its low X-ray absorption and its good mechanical strength. With this design, the quantity of electrolyte around the sample is very small, so that the X-ray absorption will be reduced as well. To reduce further the attenuation, the energy was also increased up to $E=18.4$ ke $\mathrm{V}$ corresponding to a wavelength $\lambda=0.0675 \mathrm{~nm}$ and leads to an acceptable transmission of $50 \%$. The sample is placed on a steel sample holder on which the electrical contacts are made. The sample is glued with indium which can be easily liquefied because of its low melting temperature $\left(T_{f}=156^{\circ} \mathrm{C}\right)$. After cooling, the sample sticks to the support and a good electrical contact is ensured. Furthermore, to avoid electrochemical reactions with the metallic support, we deposited a silicon seal on the support and around the sample. Thus, the entire surface of the sample on the $360^{\circ}$ can be probed by the X-ray beam at grazing incidence. Samples were anodized in this electrochemical cell under constant voltage with an acid electrolyte at constant temperature using a potentiostat Parstat 2273 (AMETEK Inc., USA) to apply the constant voltage and follow in situ the I-V curve.

\section{Results and Discussion}

Several conditions of anodization were investigated by means of in situ GISAXS. Here, we report on results obtained on one sample issued from a single anodization, two from a double anodization, and one from a pretextured sample.

3.1. Single Anodization. An aluminum layer is anodized in phosphoric acid ( $3 \mathrm{wt} \%)$ at a voltage of $90 \mathrm{~V}$. GISAXS images were taken every $5.22 \mathrm{~s}$, and a video was recorded during 
the in situ anodization (Supplementary 1). Four relevant GISAXS images taken at $t=0,502,1046$, and $4000 \mathrm{~s}$ are presented in Figure 3.

During the first stages of anodization, no scattering from the alumina pores is observed. From $300-400$ s, broad spots originating from the pores scattering gradually appear on each side of the beam stop, as can be seen in Figure 3(a) and the video. The intensity of the scattering signal increases with time (Figures 3(b) and 3(c)). The observed pattern is typical of an array of relative constant interobject distance but with a poor organization [14]. We calculated the average interpore distance to be $210 \mathrm{~nm}$, which is close to the expected value $(225 \mathrm{~nm})$ for the applied voltage. Furthermore, by observing the evolution of the scattering spots with time, we actually follow the growth of the porous alumina. The signature of the pores first appears in the GISAXS images at $300-400 \mathrm{~s}$, and with increasing anodization time, the spots become brighter corresponding to an increase of the nanoporous alumina thickness. In Figure 4, we present the anodization current versus time. Stage I is described as the beginning of the oxidation: the thickness of bulk alumina increases (known as the creation of an aluminum oxide barrier layer), resulting in a current decrease. Stage II corresponds to the creation of small penetration paths in the alumina barrier layer by the local dissolution of alumina by the acid creating embryos of cavities. Stage III corresponds to the cylindrical pore formation. The equilibrium between oxidation and dissolution is reached at stage IV leading to the growth of cylindrical pores in depth with a constant aluminum oxide barrier layer thickness.

To quantify the evolution of the observed GISAXS peaks, the integrated intensity of the left spot along $q_{y}$ is measured. The values are plotted with the anodization time in Figure 4 . The integrated intensity linearly increases with anodization time with a breaking slope close to $t=400 \mathrm{~s}$. At this time, a clear spot appears with a measurable FWHM which corresponds to the first point of the FWHM curve, corresponding to the beginning of stage III during which the pore formation increases gradually. The global porosity of the layer increases, leading to the increasing of the integrated intensity. During this stage III, the pore shape quality increases and also the distribution decreases leading to a FWHM decrease. Finally, stage IV corresponds to a permanent regime (the FWHM is constant) showing that the cylindrical pores are formed and propagate in depth. This leads to the porosity increasing and so to the intensity increasing.

3.2. Double Anodization. A first sample was anodized at $V=40 \mathrm{~V}$ in oxalic acid, and the electrochemical evolution of the aluminum layer was followed with GISAXS recording (Supplementary 2). Four images for this sample are presented in Figure 5. We first observe $(t=0$ Figure 5(a)) only a scattering halo around the direct X-ray beam indicating an X-ray scattering from a rough surface. This roughness corresponds to the imprint of the bottom of the pores of the first anodization. With increasing the time of anodization, scattering rods appear on both sides of the beam stop just before $t=50 \mathrm{~s}$. These rods are elongated along the $q_{z}$ axis and originated from a scattering layer present at the

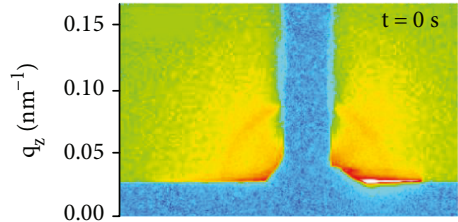

(a)

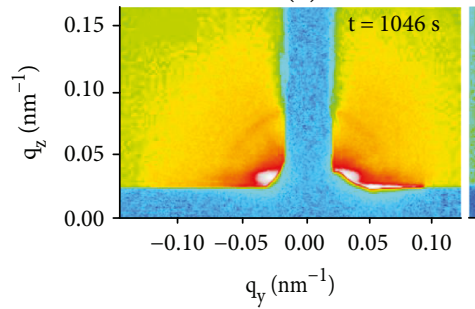

(c)

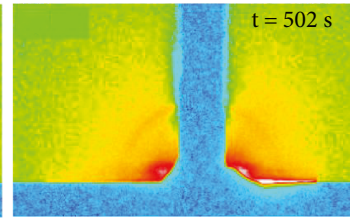

(b)

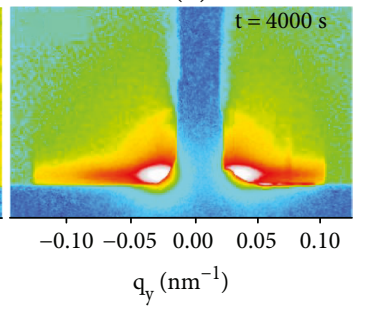

(d)
FIGURE 3: GISAXS images during a single anodization under $90 \mathrm{~V}$ with phosphoric acid (3 wt\%) at (a) $0 \mathrm{~s}$, (b) $502 \mathrm{~s}$, (c) $1046 \mathrm{~s}$, and (d) $4000 \mathrm{~s}$. A video of the GISAXS images was recorded during the anodization (Supplementary 1).

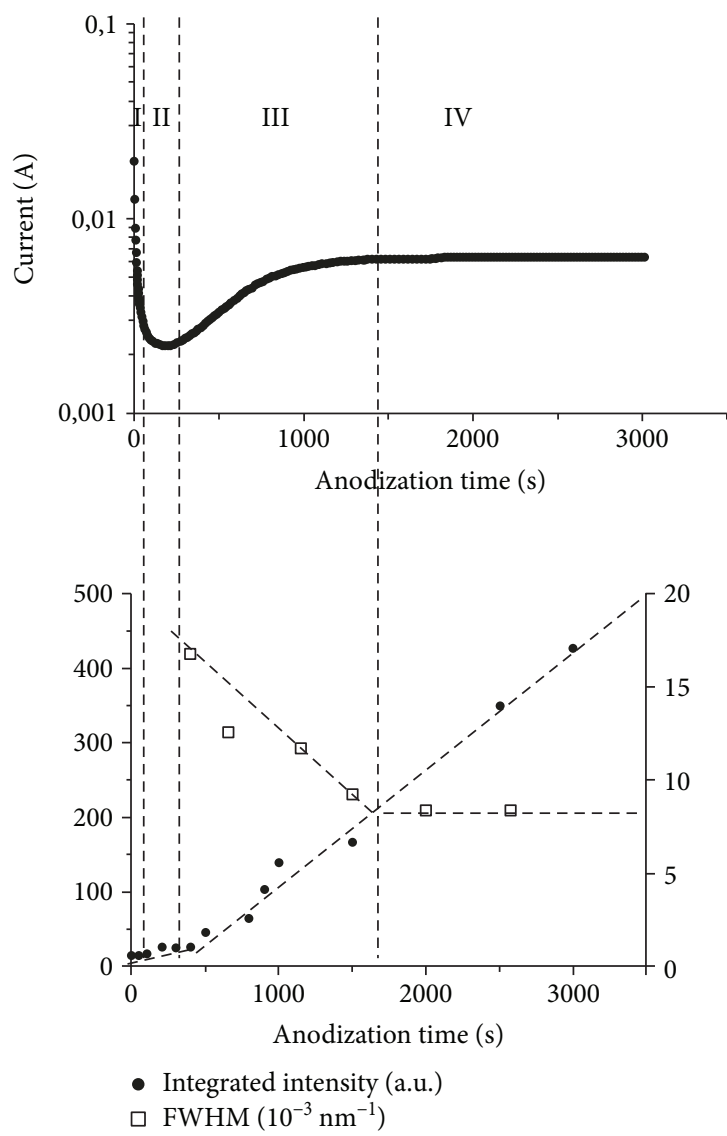

FIgURE 4: Anodization of an aluminum layer in phosphoric acid (3 wt\%) under a voltage of $90 \mathrm{~V}$. (a) Anodization current versus time (stage II appears around $t=100 \mathrm{~s}$, stage III (pore formation) at around $t=400 \mathrm{~s}$, and stage IV (pore propagation) around $t=$ $1700 \mathrm{~s}$ ). (b) Integrated intensity along the $q_{y}$ axis and full width at half maximum (along $q_{y}$ axis) as a function of the anodizing time. 


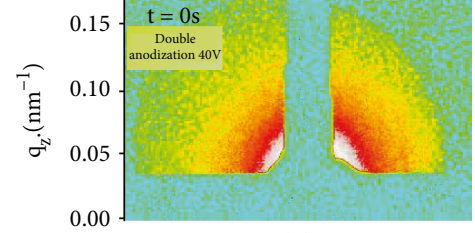

(a)

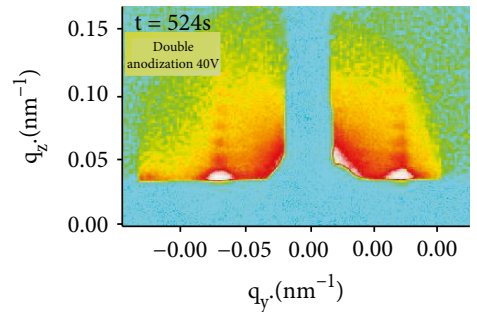

(c)

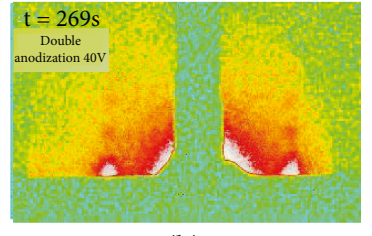

(b)

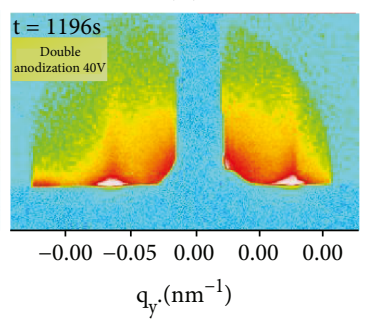

(d)
FIGURE 5: GISAXS images taken during the second anodization of a double anodization process for a sample anodized in $3 \mathrm{wt} \%$ oxalic acid at $40 \mathrm{~V}$ at (a) $0 \mathrm{~s}$, (b) $269 \mathrm{~s}$, (c) $524 \mathrm{~s}$, and (d) $1196 \mathrm{~s}$ (Supplementary 2). Another video is available for a sample anodized at $90 \mathrm{~V}$ in phosphoric acid (Supplementary 3).

sample surface. This corresponds to the initiation of the pore's formation.

A similar behavior is observed for a sample anodized at $V=90 \mathrm{~V}$ in phosphoric acid (Supplementary 3). In that case, broad rods exist at $t=0 \mathrm{~s}$ due to the patterned surface and then pores appear at around $t=500 \mathrm{~s}$ as evidenced by modulations on the rods along the $q_{z}$ direction. For the sample anodized at $40 \mathrm{~V}$ in oxalic acid, these modulations appear at around $140 \mathrm{~s}$. The difference between both times of pore initiation is due to experimental conditions: the kinetics increases with voltage, but at the same time, it is clearly slower with phosphoric acid than with oxalic acid. The GISAXS pattern indicates that this porous layer is quite homogeneous and corresponds to the existence of regular pores. When the anodization process progresses, the period of the thickness fringes decreases, and for a very long time, they are no more visible since the fringes are too close to be resolved. The current-voltage (I-V) curve for the $40 \mathrm{~V}$ anodization in oxalic acid is plotted in Figure 6. The four regimes of anodization can be distinguished as in Figure 4(a), here with regime III starting at around $50 \mathrm{~s}$ and regime IV at around $100 \mathrm{~s}$. The pore apparition at $50 \mathrm{~s}$ is in agreement with the video observation. The layer thickness was also measured from the thickness fringes period, and its linear evolution is reported on the right axis of Figure 6.

The equation from the linear regression of the experimental data is estimated as thickness $(\mathrm{nm})=1.015 t(\mathrm{~s})-38.382$. For comparison, a sample at around $1000 \mathrm{~s}$ of anodization was measured ex situ with SEM imaging. The corresponding thickness is reported in Figure 6 with a cross and fit well with the GISAXS observations. The slope is linear until the early stages of anodization, showing that the pore growth is nearly constant for these times. These very short times are very difficult to be observed ex situ by SEM, which require numerous samples (one sample for one anodization time).

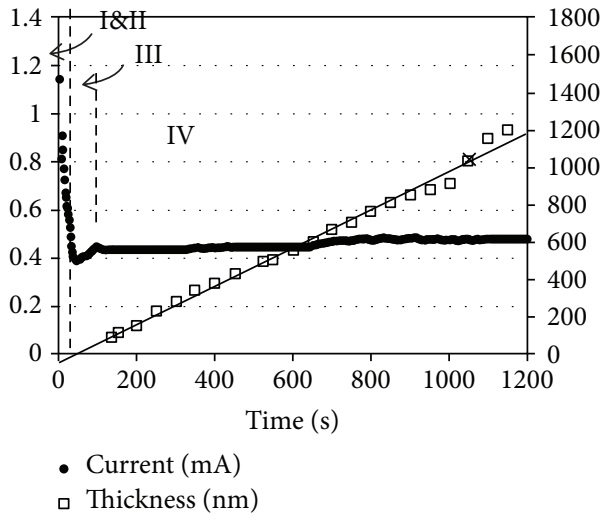

FIgUre 6: Samples anodized under $40 \mathrm{~V}$ using oxalic acid at $3 \mathrm{wt} \%$ (second anodization of a double anodization process): in situ measured current of anodization versus time under $40 \mathrm{~V}$ (left axis) and thickness of the porous layer measured in situ via the thickness fringes (right axis). The cross corresponds to the thickness of one sample measured ex situ by SEM.

Consequently, SEM observations from $t=1000 \mathrm{~s}$ down to $0 \mathrm{~s}$ is not the best adapted technique. In situ GISAXS measurements are a more appropriate method. The negative value of the origin coordinate $(-38.382)$ shows a nonlinearity of the line close to $t=0 \mathrm{~s}$. This evidences the nonlinearity of the pore growth at the beginning of the anodization. This can be seen as incubation time before the pore growth, including regime I with the oxide barrier growth and regime II with the apparition of small penetration paths in the oxide barrier layer. The straight line intersection with time axis at around $t=50 \mathrm{~s}$ exactly corresponds to the pore growth beginning of regime III.

3.3. Nanoimprinted Samples. Finally, the anodization with a pretextured sample is investigated. A sample of $0.5 \times 0.5 \mathrm{~cm}^{2}$ with $1 \mu \mathrm{m}$-thick Al layer was etched on its entire surface by a hexagonal lattice of small holes with a period $a=100 \mathrm{~nm}$. Unlike the other samples, it is necessary to orient the sample in the GISAXS setup before anodization. We orient the sample along the inplane (100) direction before anodization. This is possible since the pretextured holes made by nanoimprint scatter the X-ray beam, giving rise to welldefined rods, as can be observed in Figure 7(a) (recorded at $t=0 \mathrm{~s}$ ). After orientation, the anodization is performed in oxalic acid at $3 \mathrm{wt} \%$, the voltage is settled at $40 \mathrm{~V}$, and the temperature is at $11^{\circ} \mathrm{C}$. We measure the scattering images every $5.22 \mathrm{~s}$ as for double and single anodization samples. Four of them are presented in Figure 7 (Supplementary 4 is also available).

As mentioned above, we can observe very well-defined rods at $t=0 \mathrm{~s}$, with a low intensity (due to the low quantity of scattering matter). On the images (b) and (c), respectively, at $t=54 \mathrm{~s}$ and $103 \mathrm{~s}$, we observe that the intensity of these rods is increasing. In addition, Kiessig fringes can be observed. As for the double anodization, they are getting closer and closer. Finally, the image (d) at $t=600 \mathrm{~s}$ was taken after the end of anodization observed on the $I(t)$ curve. The 


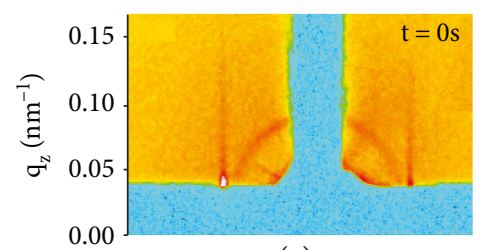

(a)

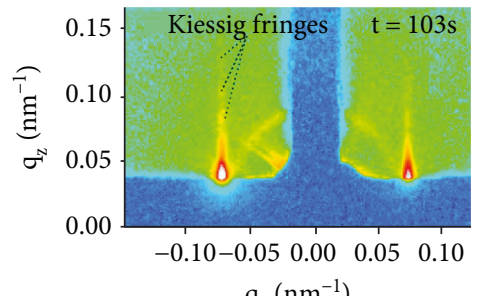

$\mathrm{q}_{\mathrm{y}}\left(\mathrm{nm}^{-1}\right)$

(c)

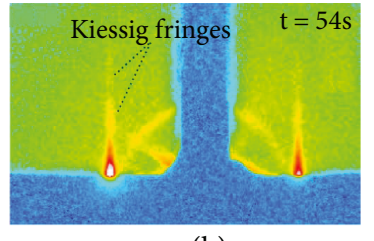

(b)

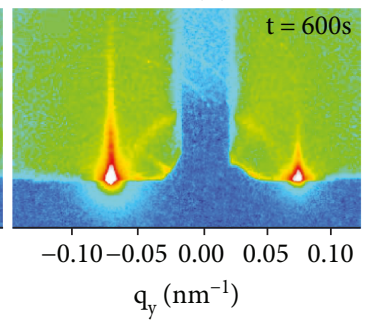

(d)
FIGURE 7: GISAXS image of a pretextured sample anodized under $40 \mathrm{~V}$ with oxalic acid at $3 \mathrm{wt} \%$ : (a) at $0 \mathrm{~s}$, (b) at $54 \mathrm{~s}$, (c) at $103 \mathrm{~s}$, and (d) at $600 \mathrm{~s}$ (Supplementary 4).

rods are very intense, and Kiessig fringes can hardly be distinguished. In comparison with the single or double anodization, the rods are very narrow and bright. This is a proof of a very high-quality inplane network of pores. A second difference is the presence of rods even at $t=0 \mathrm{~s}$, coming from the scattering of the pattern engraved with NIL. Except for these first stages of anodization, evolution of the scattering images is quite similar. As for the double anodization process, we study the evolution of the period of the Kiessig fringes. We perform these measurements up to $250 \mathrm{~s}$ since after that, the fringes are no more distinguishable. Results are shown in Figure 8. The period of the Kiessig fringes (Figure 8(a)) is related to the nanoporous alumina layer thickness thus to the depth of the cylindrical pores. As soon as those fringes appear, we can accurately measure the length of the pores and the evolution of the nanoporous alumina layer thickness with time. The rods cross section along $q_{z}$ is presented in Figure 8. With the interfringes' distance $\Delta q_{z}$, the thickness $h$ can be estimated as follows:

$$
h=\frac{2 \pi}{\Delta q_{z}} .
$$

We measure $q_{z}=0.004 \pm 5.10^{-4} \mathrm{~nm}^{-1}$ corresponding to $h=1571 \pm 160 \mathrm{~nm}$, close to the local value measured by SEM of $1620 \mathrm{~nm}$. We also observe large fringes which are produced by a thin alumina layer, the so-called "barrier layer," existing at the bottom of the pores. This barrier layer determines pore's dimensions: it forms the walls between two pores and is present at the bottom of the pores. With a closer look at the bottom of the pores, the barrier layer has a half circle shape with a thickness $t_{b}$. We measure $\Delta q_{z}=$ $0.064 \mathrm{~nm}^{-1}$ which corresponds to $t_{b}=98 \mathrm{~nm}$. This corresponds to the expected value from the electrochemical parameters used for these experiments.
As for the double anodization presented above, we observe a strict linear behavior during stage IV of anodization. The comparison from SEM observation (cross symbol) is reported for an ex situ sample at longer anodization time and is in agreement with X-ray results. For shorter times of anodization $(t<600 \mathrm{~s})$, in situ GISAXS observations provide measurements useful for the knowledge of the early stages of pore formation. We observe that the slope is higher than that for the double anodization performed under the same conditions: coefficient of 2.67, as compared to 1.02 for the double anodization. This might just come from the temperature of the electrolyte during the anodization; indeed, the sample was anodized at a temperature of $11^{\circ} \mathrm{C}$ against a temperature of $5^{\circ} \mathrm{C}$ for the double anodization. It is therefore natural that the speed is much higher. In addition, the origin coordinate at $t=0 \mathrm{~s}$ is $+9.4 \mathrm{~nm}$ and not a negative value as it was the case with the double anodization. By projection of the linear regression for the early stage of anodization, this confirms that the pores have a nonzero depth at the beginning of the anodization process. The value of $9.4 \mathrm{~nm}$ would then correspond to the depth of the holes made by the pretexturing process (nanoimprinting and etching). This value is consistent with the plasma etching: a $10 \mathrm{~nm}$ etching depth is expected [11]. Once again, if we assume that we have a linear behavior at the earlier stage of anodization, we find coherent values of depth of the patterned holes with the plasma etching calibration. The difference with the double anodization would concern stages I, II, and III instead of only stage III, which means the pores grow from the beginning of the anodization.

The pore enlargement of the same sample was also observed in situ and is available in Supplementary 5. We first observed a fringe contrast increase up to around $2000 \mathrm{~s}$ corresponding to an increased density contrast between pores and alumina, which mean the pore diameter increasing and also the quality improvement of the cylindrical shape. Then after an optimal pore/alumina contrast value around $2000 \mathrm{~s}$, we observe a decreasing in the fringe contrast. A clear degradation of the signal is finally observed from roughly $3000 \mathrm{~s}$ evidencing a global degradation of the structure (alumina wall perforation and global losses of matter). The rods disappeared at around $4000 \mathrm{~s}$, when the layer disappears, and finally, the GISAXS signal completely disappears at around $t=4500 \mathrm{~s}$ corresponding to the total debonding of the alumina layer.

3.4. Inplane Reconstruction and Pore Densification. By recording the GISAXS patterns at various azimuthal angles $\mu$, we could reconstruct inplane reciprocal space maps for a single and a double anodization samples. As mentioned in the above sections, for these types of samples, the GISAXS patterns are unchanged with $\mu$ for these samples. This means that the pore organization is isotropic in the plane of the sample surface. This is in good agreement with the fact that only a local organization is observed for single anodization with a rather high disorder, whereas for a double anodization, a hexagonal organization in small domains $(<1 \mu \mathrm{m})$ is observed with an inplane randomly orientated distribution of the domains. On the other hand, pretextured samples reveal a scattering pattern strongly dependent with the $\mu$ 


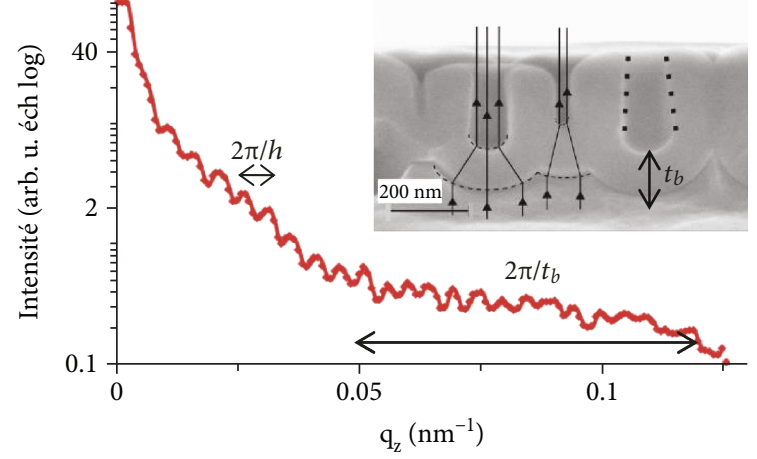

(a)

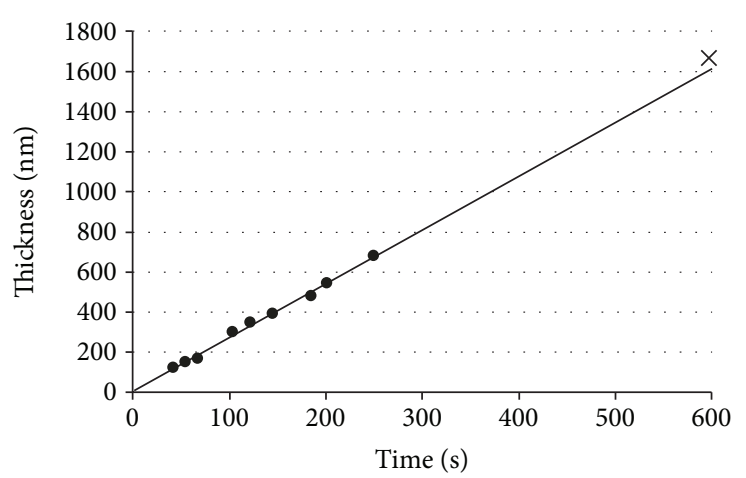

(b)

FIGURE 8: Samples anodized at $40 \mathrm{~V}$ using oxalic acid (3 wt\%) with pretextured sample. (a) Cross section along $q_{z}$ direction from the scattering rod. The inset shows the corresponding SEM cross section with the schematically representation of the electrical field lines. (b) Porous layer thickness measured from the Kiessig fringes at the early stages of formation. The comparison from SEM observation (cross symbol) is reported for an ex situ sample at longer anodization time. The linear regression equation is thickness $(\mathrm{nm})=2.7 t(\mathrm{~s})+9.4$.

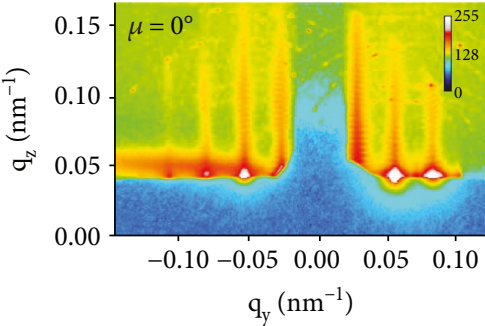

(a)

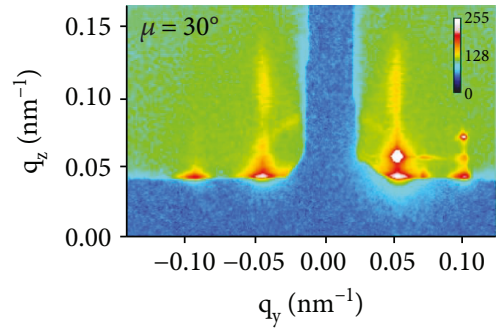

(b)

FIGURE 9: GISAXS images in logarithmic scale of a pretextured sample with a mould period of $250 \mathrm{~nm}$ and anodized at $100 \mathrm{~V}$. Images were recorded at azimuthal angles $\mu$ of (a) $0^{\circ}$ corresponding to the (100) direction and (b) $30^{\circ}$ corresponding to the (110) direction.

orientation. For example, for $\mu=0^{\circ}$, we acquired the GISAXS image of Figure 9(a). The observed scattering rods show modulations along the $q_{z}$ direction corresponding to thickness fringes as previously presented in Figure 8(a). From the period of these fringes, a thickness of $1571 \mathrm{~nm}$ is estimated in agreement with an expected value of $1620 \mathrm{~nm}$ measured by SEM. Similar rods are observed for $\mu=30^{\circ}$ which confirms the hexagonal structure with a long range distance.

By acquiring images at several azimuthal angles $(\mu)$, it is possible to reconstruct the inplane reciprocal space map as presented in Figures 10(b) and 10(c). At each step in $\mu$, we extract a region of interest (ROI) of the image, and then the $\left(q_{\mathrm{y}}, \mu\right)$ polar coordinates of each pixel are transformed in Cartesian coordinates related to the sample $\left(q_{x}^{\text {sample }}=q_{y} \cos (\mu)\right.$, $\left.q_{y}^{\text {sample }}=q_{y} \sin (\mu)\right)$, and the corresponding intensity is plotted following the color scale at the corresponding position. This representation was applied to a pretextured sample with a $100 \mathrm{~V}$ anodization voltage. The SEM image (Figure 10(a)) shows a regular quasihexagonal pore lattice. Figure 10(b) shows the reciprocal space reconstruction of the pretextured sample of Figure 10(a). The mapping reveals very thin scattering spots on a hexagonal lattice, giving evidence of the long range order of the hexagonal pore structure. The scattering signal is elongated along the radial direction, due to the shape of the beam. The reciprocal lattice is characterized by $a^{*}=b^{*}=0.03 \mathrm{~nm}^{-1}$ and $\gamma=53.2^{\circ}$ that correspond in real space to a rhombohedral unit cell with $a=b=261.5 \mathrm{~nm}$ and $\gamma=126.8^{\circ}$.

This result is in agreement with the value obtained from the SEM images $(264 \mathrm{~nm})$. The lattice distortion is due to the initial shape of the nanoimprint mould where a perfect equilateral triangle of the basic lattice was not achieved. Similar measurements and reconstructions (not shown here) have been performed with the perfect hexagonal array $(b=100 \mathrm{~nm}$ with SEM) and confirm the nondistortion of the lattice for this sample $\left(a=b=102 \mathrm{~nm}, \gamma=60^{\circ}\right)$.

A SEM image of a sample anodized according to the density multiplication conditions described elsewhere [16] is presented in Figure 10(c). We observed an assembly of pores well organized in hexagonal arrays. With a more careful look at the surface, we observed two families of pores: prepatterned pores with a circular cross section and induced pores with a triangular cross section ("up" and "down" triangles). Self-organized-induced pores are observed exactly in between three imprinted pores, matching the predicted hexagonal arrangement. Figure 10(d) shows the reciprocal space reconstruction of the pretextured sample shown in Figure 10(c). This map is very similar to the one of Figure 10(b). We achieved an accurate measurement of 


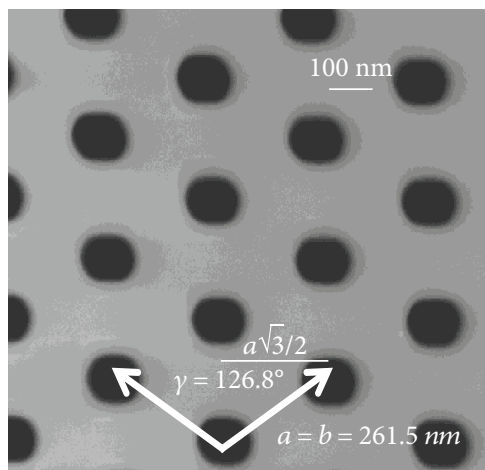

(a)

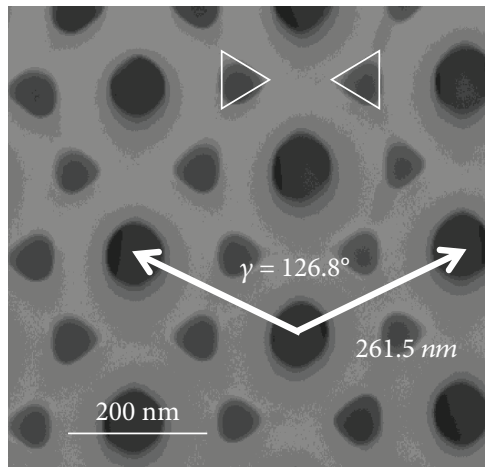

(c)

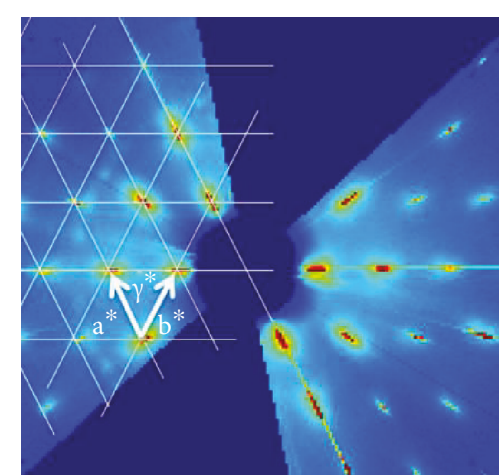

(b)

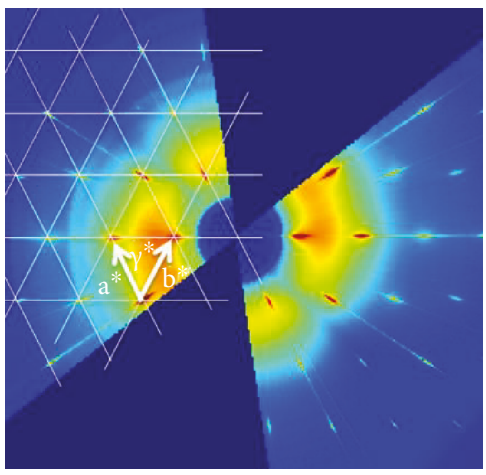

(d)

FIgURE 10: Pretextured sample with a $250 \mathrm{~nm}$ step mould (a, c) plane view SEM images and (b, d) inplane reciprocal space maps reconstruct with the acquisition of images at several azimuthal angles $(\mu)$. The sample was anodized at $(a, b) 100 \mathrm{~V}$ and $(c, d) 65 \mathrm{~V}$.

the intensity of the spots. However, no modulation of the intensity was observed, whereas it was expected due to the presence of induced pores. This could be due to a too low dynamic between the GISAXS signal and the background noise level.

\section{Conclusions}

We successfully performed nondestructive in situ GISAXS measurements during aluminum anodization. We designed an electrochemical cell which allows recording scattering images of porous alumina during the anodization for all azimuthal angles $\left(360^{\circ}\right)$ and with a low X-ray absorption. The observed thickness fringes allow tracking the thickness of the nanoporous layer and the pores length within the same sample, which is not possible using standard scanning electron microscopy. We observed the similarity in the pore growth between a single, a double, or a pretextured anodization, in the permanent regime so-called stage IV, with linear time dependences. A special attention was paid to the early stages of formation at very short times. For the single and double anodization, our studies suggest that pores appear only at stage III and then grow linearly, but with an incubation delay before stage III due probably to the pore initiation. The case of prepatterned sample suggests a different behavior due to the presence of the holes before anodization.

\section{Data Availability}

The data used to support the findings of this study are available from the corresponding author upon request.

\section{Conflicts of Interest}

All authors declare that there is no competing interest.

\section{Authors' Contributions}

The authors declare that all authors have individually contributed to this work.

\section{Acknowledgments}

We acknowledge the French region Rhône-Alpes for funding this project.

\section{Supplementary Materials}

Supplementary 1. Video single anodization: GISAXS video during a single anodization under $90 \mathrm{~V}$ with phosphoric acid (3 wt\%).

Supplementary 2. Video double anodization $40 \mathrm{~V}$ : GISAXS video during the second anodization of a double anodization process for a sample anodized in $3 \mathrm{wt} \%$ oxalic acid at $40 \mathrm{~V}$. 
Supplementary 3. Video double anodization $90 \mathrm{~V}$ : GISAXS video during the second anodization of a double anodization process for a sample anodized in $3 \mathrm{wt} \%$ oxalic acid at $90 \mathrm{~V}$.

Supplementary 4. Video pretextured sample anodized at $40 \mathrm{~V}$ : GISAXS video during the anodization of a pretextured sample anodized under $40 \mathrm{~V}$ with oxalic acid at $3 \mathrm{wt} \%$.

Supplementary 5. Video enlargement: the pore enlargement of the pretextured sample after anodization at $40 \mathrm{~V}$ was observed in situ.

\section{References}

[1] H. Oshima, H. Kikuchi, H. Nakao et al., "Detecting dynamic signals of ideally ordered nanohole patterned disk media fabricated using nanoimprint lithography," Applied Physics Letters, vol. 91, no. 2, article 022508, 2007.

[2] H. Liu, B. Lu, S. Wei, M. Bao, Y. Wen, and F. Wang, "Electrodeposited highly-ordered manganese oxide nanowire arrays for supercapacitors," Solid State Sciences, vol. 14, no. 7, pp. 789-793, 2012.

[3] D. Buttard, L. Dupré, T. Bernardin, M. Zelsmann, D. Peyrade, and P. Gentile, "Confined growth of silicon nanowires as a possible process for third generation solar cells," Physica Status Solidi (c), vol. 8, no. 3, pp. 812-815, 2011.

[4] F. Keller, M. S. Hunter, and D. L. Robinson, "Structural features of oxide coatings on aluminum," Journal of the Electrochemical Society, vol. 100, no. 9, pp. 411-419, 1953.

[5] H. Masuda and K. Fukuda, "Ordered metal nanohole arrays made by a two-step replication of honeycomb structures of anodic alumina," Science, vol. 268, no. 5216, pp. 1466-1468, 1995.

[6] O. Jessensky, F. Muller, and U. Gosele, "Self-organized formation of hexagonal pore arrays in anodic alumina," Applied Physics Letters, vol. 72, no. 10, pp. 1173-1175, 1998.

[7] J. Byun, J. I. Lee, S. Kwon, G. Jeon, and J. K. Kim, "Highly ordered nanoporous alumina on conducting substrates with adhesion enhanced by surface modification: universal templates for ultrahigh-density arrays of nanorods," Advanced Materials, vol. 22, no. 18, pp. 2028-2032, 2010.

[8] C. Y. Liu, A. Datta, N. W. Liu, C. Y. Peng, and Y. L. Wang, "Order-disorder transition of anodic alumina nanochannel arrays grown under the guidance of focused-ion-beam patterning," Applied Physics Letters, vol. 84, no. 14, pp. 25092511, 2004.

[9] Z. Sun and H. K. Kim, "Growth of ordered, single-domain, alumina nanopore arrays with holographically patterned aluminum films," Applied Physics Letters, vol. 81, no. 18, pp. 3458-3460, 2002

[10] B. Kim, S. Park, T. J. McCarthy, and T. P. Russell, "Fabrication of ordered anodic aluminum oxide using a solvent-induced array of block-copolymer micelles," Small, vol. 3, no. 11, pp. 1869-1872, 2007.

[11] W. Lee, H. Han, A. Lotnyk et al., "Individually addressable epitaxial ferroelectric nanocapacitor arrays with near $\mathrm{Tb}$ inch $^{-2}$ density," Nature Nanotechnology, vol. 3, no. 7, pp. 402-407, 2008.
[12] K.-L. Lai, M.-H. Hon, and I.-C. Leu, "Fabrication of ordered nanoporous anodic alumina prepatterned by mold-assisted chemical etching," Nanoscale Research Letters, vol. 6, no. 1, p. 157, 2011.

[13] S. Fournier-Bidoz, V. Kitaev, D. Routkevitch, I. Manners, and G. A. Ozin, "Highly ordered nanosphere imprinted nanochannel alumina (NINA)," Advanced Materials, vol. 16, no. 23-24, pp. 2193-2196, 2004.

[14] H. Masuda, H. Yamada, M. Satoh, H. Asoh, M. Nakao, and T. Tamamura, "Highly ordered nanochannel-array architecture in anodic alumina," Applied Physics Letters, vol. 71, no. 19, pp. 2770-2772, 1997.

[15] T. S. Kustandi, W. W. Loh, H. Gao, and H. Y. Low, "Waferscale near-perfect ordered porous alumina on substrates by step and flash imprint lithography," ACS Nano, vol. 4, no. 5, pp. 2561-2568, 2010.

[16] T. Gorisse, L. Dupré, P. Gentile, M. Martin, M. Zelsmann, and D. Buttard, "Highly organised and dense vertical silicon nanowire arrays grown in porous alumina template on $<100\rangle$ silicon wafers," Nanoscale Research Letters, vol. 8, no. 1, pp. 287-294, 2013.

[17] M. E. Nasir, D. W. E. Allsopp, C. R. Bowen, G. Hubbard, and K. P. Parsons, "The fabrication of mono-domain highly ordered nanoporous alumina on a wafer scale by a guided electric field," Nanotechnology, vol. 21, no. 10, p. 105303, 2010.

[18] G. Renaud, R. Lazzari, and F. Leroy, "Probing surface and interface morphology with grazing incidence small angle X-ray scattering," Surface Science Reports, vol. 64, no. 8, pp. 255-380, 2009.

[19] E. Vlieg, "A (2+3)-type surface diffractometer: mergence of the Z-axis and (2+2)-type geometries," Journal of Applied Crystallography, vol. 31, no. 2, pp. 198-203, 1998. 


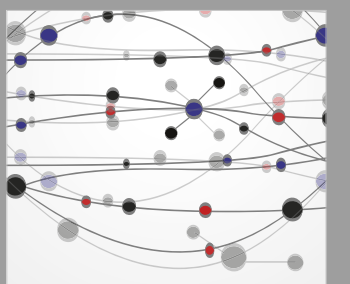

The Scientific World Journal
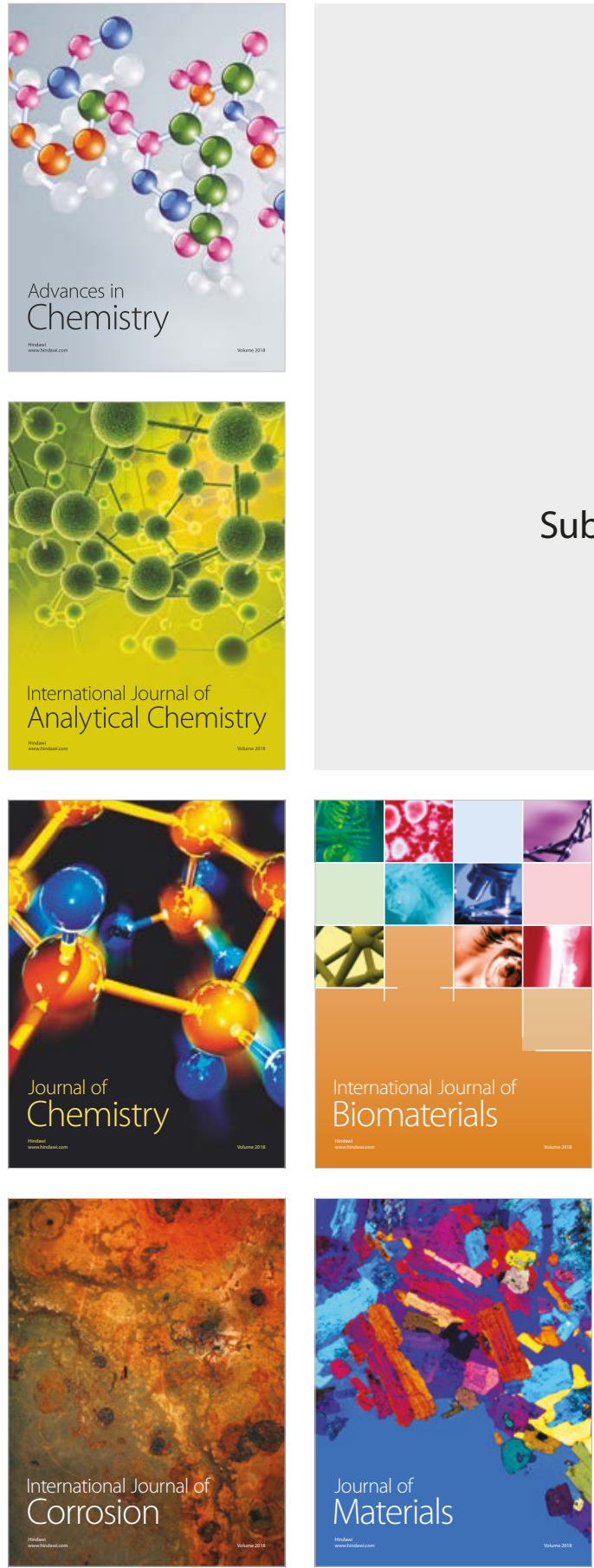

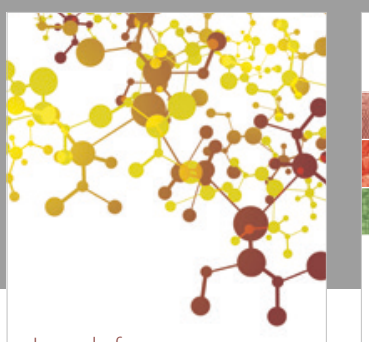

Journal of

Applied Chemistry
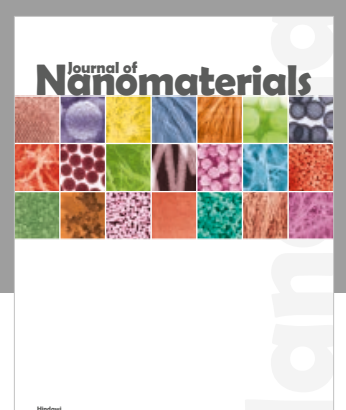

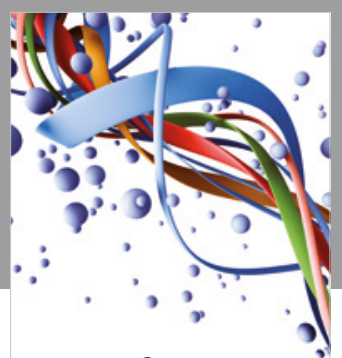

Scientifica

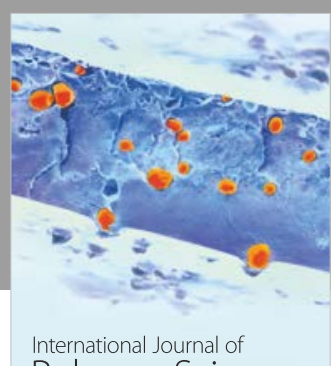

Polymer Science

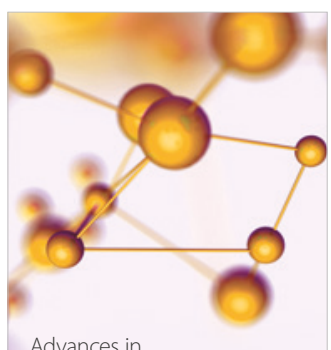

Physical Chemistry
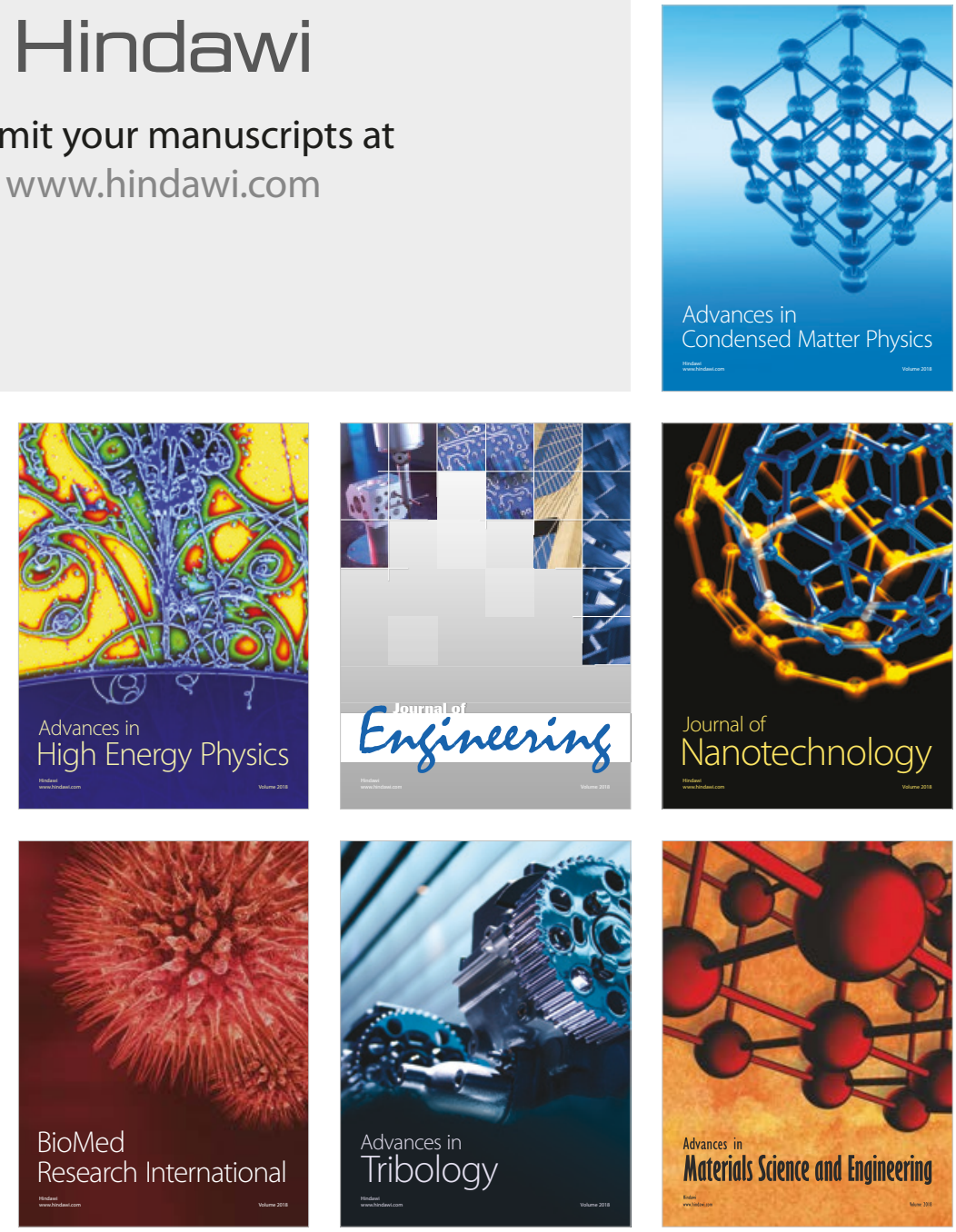